CASO CLÍNICO

\title{
Sangrado colónico secundario a migración de un over the scope clip
}

\author{
Wagner Enrique Ramírez-Quesada*, Daniela Grant-Alpizar, Óscar González-Pacheco, Pablo Coste-Murillo \\ y Gerardo Avendaño-Alvarado
}

Servicio de Gastroenterología, Hospital Rafael Ángel Calderón Guardia, San José, Costa Rica

\section{Resumen}

Los over the scope clips (OTSC) son dispositivos endoscópicos con capacidad de cierre transmural utilizados en diversos escenarios clínicos, como sangrado digestivo, perforaciones, fístulas o fugas, todos con variadas tasas de éxito pero en general con resultados positivos. Su uso ha venido a complementar las herramientas del endoscopista terapéutico debido a la mayor tasa de complicaciones de las nuevas técnicas endoscópicas. No obstante, pese a ser herramientas con gran versatilidad no están exentos de efectos adversos potencialmente graves. A continuación presentamos el caso de una paciente en quien la aplicación de un OTSC desecadenó un sangrado colónico masivo que ameritó extracción endoscópica del dispositivo.

Palabras clave: OTSC. Efecto adverso. Sangrado digestivo bajo. Hemorragia colónica. Fuga esofágica.

\section{Colonic bleeding secondary to over the scope clip migration}

\begin{abstract}
The over the scope clips (OTSC) technology are endoscopic tools with the capacity for a transmural closure used in various clinical scenarios, such as gastrointestinal bleeding, perforations, fistulae or leaks, each one with different success rates but with positive results in general. Their use has complemented the therapeutic endoscopist armamentarium due to the increased complications of the new endoscopic techniques. Even though it has great versatility, they are not exempt of potentially serious adverse effects. We present the case of a patient in which an OTSC provoked a massive colonic hemorrhage that needed endoscopic extraction of the clip.
\end{abstract}

Key words: OTSC. Adverse effect. Lower GI bleed. Colonic hemorrhage. Esophageal leak.

\section{Correspondencia:}

*Wagner Enrique Ramírez-Quesada

Servicio de Gastroenterología

Hospital Rafael Ángel Calderón Guardia

Av. 7, calle 17

Fecha de recepción: 26-11-2018

Fecha de aceptación: 12-12-2018

San José, Costa Rica

E-mail: wagner.ramirezquesada@gmail.com
Disponible en internet: 05-02-2019 Endoscopia. 2019;31:47-49 www.endoscopia-ameg.com 0188-9893/O 2018. Asociación Mexicana de Endoscopia Gastrointestinal, publicado por Permanyer México SA de CV, todos los derechos reservados. 


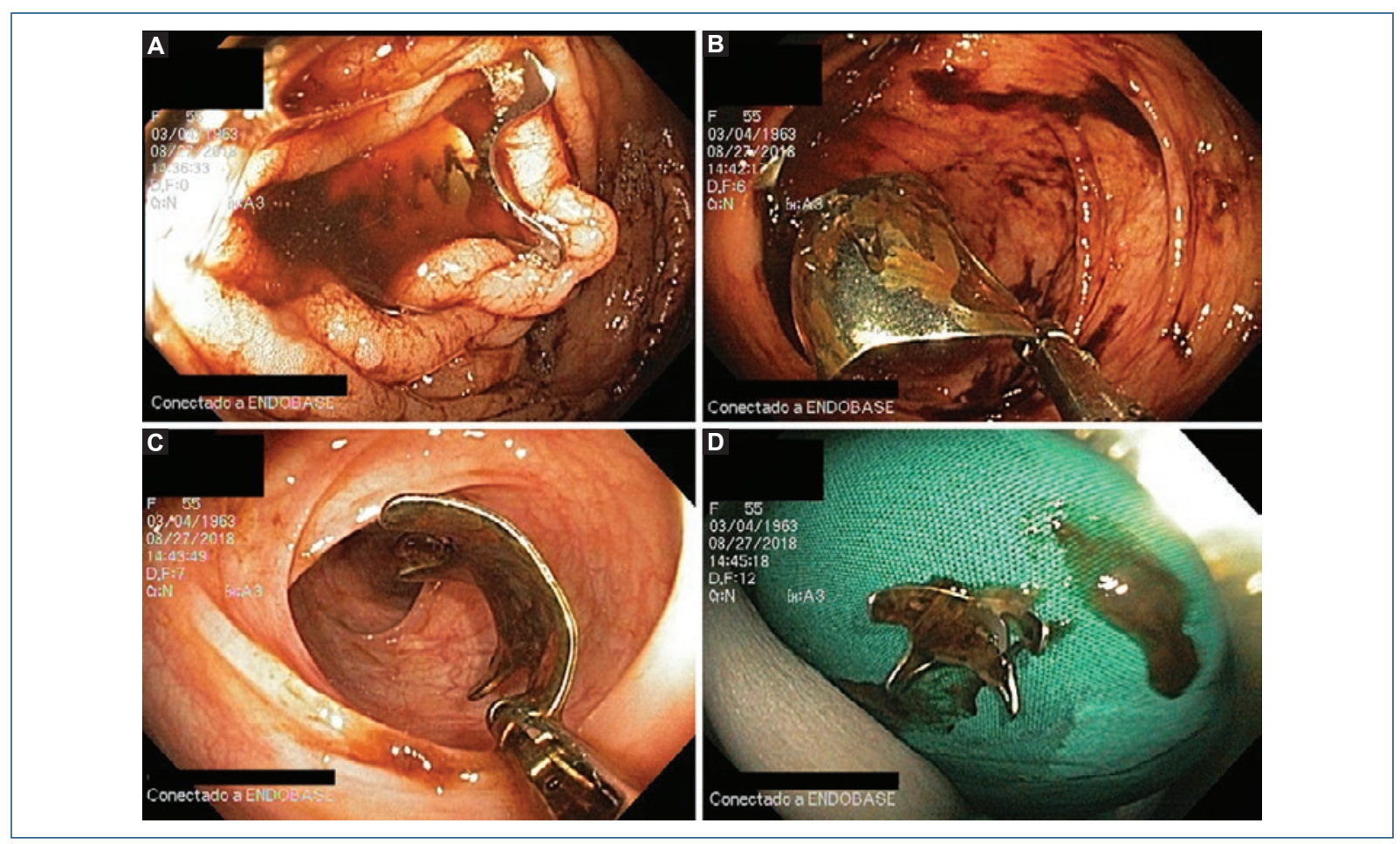

Figura 1. (A) OTSC enclavado en orificio apendicular con múltiples erosiones y restos hemáticos alrededor. (B) Movilización inicial del OTSC luego de dislocarlo del orificio apendicular. (C) Extracción exitosa del OTSC a través del colon. (D) OTSC extraído por completo del paciente. OTSC: over the scope clip.

\section{Introducción}

Los over the scope clips (OTSC; Ovesco Endoscopy, Tübingen, Alemania) forman parte de un sistema que permite el cierre de defectos de gran tamaño, fuentes de sangrado no variceal, perforaciones, fístulas y dehiscencias anastomóticas ${ }^{1,2}$. Están hechos a base de nitinol, lo que les confiere gran resistencia pero a la vez gran maleabilidad, esto permite al material conservar memoria y producir una gran fuerza de cierre.

\section{Usos clínicos}

Los OTSC son de gran utilidad en el contexto del sangrado digestivo, con tasas de éxito como terapia primaria de hasta un $100 \%$ y para tratamiento del resangrado con un éxito del $10-20 \%$, según la serie ${ }^{1,2}$. Su efectividad en el cierre de fugas y fístulas varía mucho si estas son agudas o crónicas. Las primeras cuentan con buenos resultados. No obstante, estudios retrospectivos han mostrado que pese a una tasa de éxito del $89 \%$ en el cierre primario de las fístulas crónicas, la recurrencia puede llegar hasta un $46 \%$ pese a buena colocación ${ }^{3,4}$.

\section{Resumen del caso}

Se presenta el caso de una paciente de 55 años, quien 11 días después de una diverticulectomía paraesofágica laparoscópica acudió al Servicio de Emergencias por un proceso séptico pleural; esto secundario a una fuga de la línea de sutura, razón por la que fue fue trasladada a otro centro en el cual se le aplicó un OTSC traumático regular con cierre exitoso de defecto luego del tratamiento médico inicial.

Cuatro meses después la paciente reingresa con otro cuadro de infección pleural, así como inicio de anticoagulación con enoxaparina sódica por una trombosis venosa profunda asociada a una tromboembolia pulmonar. Durante su estancia es valorada por rectorragia masiva y descenso abrupto de la hemoglobina hasta $4.4 \mathrm{~g} / \mathrm{dl}$.

Se realizó esofagogastroduodenoscopia sin evidencia de sangrado y se documentó en tercio distal de esófago una formación pseudodiverticular con dos fístulas maduras. Se realizó preparación intestinal rápida y se programó para colonosocopia, en la que se evidenciaron abundantes restos hemáticos. Se ingresó a la válvula ileocecal, que mostró un íleon sin trazos de 
sangre. En el ciego se evidenció un coágulo gigante que se retiró con aspiración y en el orificio apendicular se observó uno de los bordes del OTSC (Fig. 1). Este se extrajo mediante pinzas de biopsia sin documentarse defecto transmural subyacente (Figs. 1B y 1C). Posteriormente a la extracción del clip, el sangrado digestivo bajo se detuvo por completo, con resolución del cuadro, y la paciente continuó el tratamiento de su fístula crónica por parte de sus médicos tratantes.

\section{Discusión}

Los OTSC cuentan con un perfil muy alto de seguridad, pero las complicaciones asociadas a su uso pueden encontrarse en hasta un $1.7 \%$ de los casos y las complicaciones severas en hasta un $0.59 \%$. Dentro de las posibles complicaciones se pueden mencionar: estenosis intraluminal, perforaciones, pinzamiento de órganos adyacentes y aumento del tamaño de defectos subyacentes, como fístulas 5 .

Las causas de sangrado digestivo bajo esperables en este caso son la colitis isquémica secundaria a sus múltiples episodios de inestabilidad hemodinámica 0 el uso de antiinflamatorios no esteroideos (AINE) para aliviar el dolor de las múltiples intervenciones quirúrgicas $^{6}$. No obstante, después de revisión bibliográfica exhaustiva, no hemos logrado encontrar otro caso en condiciones similares donde la causa directa del sangrado fuera una erosión provocada por un OTSC enclavado en ciego.

A manera de conclusión, consideramos que la utilidad y la experiencia con los OTSC es amplia y estos cuentan con gran cantidad de literatura que los respalda, con una pequeña proporción de efectos adversos que deben tomarse en cuenta en el contexto de cada paciente. Por tanto, pese a su disponibilidad y eventual uso, debe individualizarse cada caso al momento de utilizarse y considerar sus potenciales efectos adversos luego de su colocación.

\section{Bilbiografía}

1. Kobara H, Mori H, Nishiyama N, Fujihara S, Okano K, Suzuki Y, et al. Overthe-scope clip system: A review of 1517 cases over 9 years. J Gastroenterol Hepatol. 2018 Aug 2. doi: 10.1111/jgh.14402. [Epub ahead of print]

2. Conigliaro R, Frazzoni M. Diagnosis and endoscopic management of digestive diseases. $1^{\text {st }}$ ed. Cham: Springer; 2017. pp.21-42.

3. Rogalski P. Endoscopic management of gastrointestinal perforations, leaks and fistulas. World J Gastroenterol. 2015;21:10542-52.

4. Law R, Wong Kee Song LM, Irani S, Baron TH. Immediate technical and delayed clinical outcome of fistula closure using an over-the-scope clip device. Surg Endosc. 2015;29:1781-6.

5. Sandhu DS, Gerke H. Su1516 Treatment failure and delayed complication due to shedding of the over-the scope clip: Experience in three cases. Gastrointest Endosc. 2015;81:AB312.

6. Pasha SF, Shergill A, Acosta RD, Chandrasekhara V, Chathadi KV, Early D, et al. The role of endoscopy in the patient with lower Gl bleeding. Gastrointest Endosc. 2014;79:875-85. 